\section{Visión Electrónica Más que un estado sólido \\ https://doi.org/10.14483/issn.2248-4728}

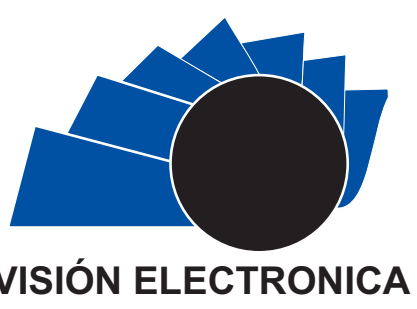

A Research Vision

\title{
Matrix of coefficients for the differential equation of Chauchy-euler
}

\author{
Matriz de coeficientes para la ecuación diferencial de Chauchy-Euler
}

Fabio Hernando Castellanos-Moreno ${ }^{1}$, Jaime Francisco Pantoja-Benavides ${ }^{2}$

\section{INFORMACIÓN DEL ARTICULO}

Historia del articulo

Enviado: 11/10/2018

Recibido: 19/11/2018

Aceptado: 30/11/2018

\section{Keywords:}

Cauchy-Euler,

Coefficients,

Differential,

Equation, Matrix,

ODEs.

\section{Palabras clave: \\ Cauchy-Euler, \\ Coeficientes, \\ Diferencial, \\ Ecuación, Matriz, \\ EDOs.}

\begin{abstract}
In this article application and advance is given to the method that allows to find in a simple way the constant coefficients that are required in the solution of a differential equation with the structure of Cauchy-Euler, presented and made known in [1]. Here we will see the construction form of the characteristic polynomials, using the aforementioned method as a basis; with a series of equations, matrices and novel methods to solve this type of ODEs and, above all, very practical for the equation of a much higher order. The idea is to present a structure or methodology in matrix form that allows to solve in a practical way differential equation of higher order with Cauchy-Euler structure.
\end{abstract}

\section{RESUMEN:}

En este artículo, se muestra una aplicación y avance al método que permite encontrar de manera simple los coeficientes constantes que se requieren en la solución de una ecuación diferencial con la estructura de Cauchy-Euler, presentada y dada a conocer en [1]. Aquí veremos la forma de construcción de los polinomios característicos, utilizando el método mencionado anteriormente como base; con una serie de ecuaciones, matrices y métodos novedosos para resolver este tipo de EDO y, sobre todo, muy prácticas para la solución de la ecuación de orden superior. La idea es presentar una estructura o metodología en forma matricial que permita resolver de manera práctica la ecuación diferencial de orden superior con la estructura de Cauchy-Euler.

\footnotetext{
${ }^{1}$ BSc. In Mathematics, Universidad Distrital Francisco José de Caldas, Colombia. BSc. In Electronic Engineering, Universidad Antonio Nariño, Colombia. MSc. In Applied Mathematics, Universidad EAFIT, Colombia. Current position: Professor at Universidad Central, Colombia. E-mail: fcastellanosm@ucentral.edu.co. ORCID: https://orcid.org/0000-0002-3905-4626.

BSc. In Mathematics, Universidad Distrital Francisco José de Caldas, Colombia. Specialist in Statistics, Universidad Nacional de Colombia, Colombia. Current position: Professor at Universidad Distrital Francisco José de Caldas, Colombia. E-mail: jfpantojab@udistrital.edu.co. ORCID: https://orcid.org/0000-0003-0107-9542.Cite this article as: F. H. Castellanos-Moreno and J. F. Pantoja-Benavides, "Matrix of coefficients for the differential equation of Cauchy-Euler", Visión electrónica, algo más que un estado sólido, vol. 1, no. 2 , Special edition, july-december 2018. DOI revista: https://doi.org/10.14483/issn.2248-4728
} 


\section{Introduction}

The differential equations are part of the mathematical modeling intended to represent and subsequently solve phenomena of reality. In this aspect, several situations can be modeled as expressions that include a variable and its respective reasons for change (successive derivatives) in a single expression. Solving these expressions is the main objective of the study of the solution of a differential equation. These equations can be classified depending on whether their coefficients are constant or variable.

When the differential equation has constant coefficients, the process to find its solution is usually simpler than if its coefficients are variable.

The Cauchy-Euler differential equations of order $\mathrm{n}$ are part of the ODE group with variable coefficients. In this case, it is difficult to obtain a solution and, in many cases, the infinite series are used, in particular, the power series to determine the solution.

However, the differential equations with the CauchyEuler structure have the peculiarity that by means of a change of variable they can be converted into equations with constant coefficients, and therefore, they can be solved.

When reducing it to an ODE with constant coefficients, there are two options:

- Apply the indeterminate coefficient method to obtain the particular solution and then undo the variable change.

- Undo the variable change and apply the parameter variation method to the Cauchy-Euler equation.

In both cases, the process becomes increasingly complex as the order of the equation increases.

\section{Differential Equation of Cauchy-Euler.}

Given the differential equation of Cauchy-Euler, in its homogeneous form

$$
t^{\mathrm{n}} \mathrm{z}^{(\mathrm{n})}+\mathrm{b}_{\mathrm{n}-1} t^{\mathrm{n}-1} \mathrm{Z}^{(\mathrm{n}-1)}+\ldots+\mathrm{b}_{0} \mathrm{z}=0
$$

Where it is done that in each term the coefficient of the $n$-th derivative of the dependent variable is the $n$-th power of the independent variable, the substitution reduces the equation (1) to a linear equation of order $n$ with constant coefficients, [2].

$\frac{d^{n} z}{d u^{n}}+c_{n-1} \frac{d^{n-1} z}{d u^{n-1}}+c_{n-2} \frac{d^{n-2} z}{d u^{n-2}}+1 / 4+c_{2} \frac{d^{2} z}{d u^{2}}+c_{1} \frac{d z}{d u}+c_{0} z=0$
This result describes a Fundamental System of Solutions of said Cauchy-Euler equation. However, finding the coefficients $c_{1}$ is not a simple process. The quick solution of (1) is known when this, is even up to order 3 and is carried to its corresponding solution of the form (2), thus,

if $t=e^{u}$ then $\operatorname{Ln}(t)=u$ with $\frac{d t}{t}=d u$ therefore

Now, as in (1) each derivative of $\mathrm{z}$ is realized with respect to $t$, using reasons of change and rule of the chain, it is obtained that $\frac{d z}{d t}=\frac{d z}{d u} \cdot \frac{d u}{d t}$ with

$$
\begin{aligned}
& \frac{d z}{d t}=\frac{1}{t} \cdot \frac{d z}{d u} \\
& \frac{d^{2} z}{d t^{2}}=\frac{1}{t^{2}}\left[\frac{d^{2} z}{d u^{2}}-\frac{d z}{d u}\right] \\
& \frac{d^{3} z}{d t^{3}}=\frac{1}{t^{3}}\left[\frac{d^{3} z}{d u^{3}}-3 \frac{d^{2} z}{d u^{2}}+2 \frac{d z}{d u}\right]
\end{aligned}
$$

Where:

$t \frac{d z}{d t}=\frac{d z}{d u} \quad t^{2} \frac{d^{2} z}{d t^{2}}=\frac{d^{2} z}{d u^{2}}-\frac{d z}{d u} \quad t^{3} \frac{d^{3} z}{d t^{3}}=\frac{d^{3} z}{d u^{3}}-3 \frac{d^{2} z}{d u^{2}}+2 \frac{d z}{d u}$

The reader can get at equations (3) to (6) easily with basic processes of differentiation and algebra, or he can also see the process solved in some books of Ordinary Differential Equations, [3]. Now, the results of (6) are replaced in the corresponding form of (1) third order

$$
t^{3} z^{(3)}+b_{2} t^{2} z^{(2)}+b_{1} t z^{\prime}+b_{0} z=0
$$

And it is gotten to $\left(\frac{d^{3} z}{d u^{3}}-3 \frac{d^{2} z}{d u^{2}}+2 \frac{d z}{d u}\right)+\mathrm{b}_{2}\left(\frac{d^{2} z}{d u^{2}}-\frac{d z}{d u}\right)+\mathrm{b}_{1}\left(\frac{d z}{d u}\right)+\mathrm{b}_{0} z=0$

then, $\quad \frac{d^{3} z}{d u^{3}}+\left(\mathrm{b}_{2}-3\right) \frac{d^{2} z}{d u^{2}}+\left(\mathrm{b}_{1}-\mathrm{b}_{2}+2\right) \frac{d z}{d u}+\mathrm{b}_{0} z=0$

This equation (9) is simply a homogeneous differential equation with constant coefficients, which can be easily solved by finding its characteristic equation, finding the roots and applying the corresponding case according to the type of roots, as seen in those cases, [4] .

\section{Proposal}

In the texts of Ordinary Differential Equations, the Cauchy-Euler equations are usually worked at most up to order 3 , since it is cumbersome the process to go from equation (1) to equation (2), as see between equations (7) to (9), the construction of equations (3) to (6) being necessary. In the article [1], for the products of the form $t^{n} \cdot \frac{d^{n} z}{d t^{n}}$, which identifies each of the terms of the 
Cauchy-Euler differential equation, proposes and demonstrates, the validity of the equation,

$$
t^{n} \frac{d^{n} z}{d t^{n}}=a_{n 1} \frac{d^{n} z}{d u^{n}}+(-1)^{1} a_{n 2} \frac{d^{n-1} z}{d u^{n-1}}+(-1)^{2} a_{n 3} \frac{d^{n-2} z}{d u^{n-2}}+\ldots \ldots+(-1)^{n-2} a_{n(n-1)} \frac{d^{2} z}{d u^{2}}+(-1)^{n-1} a_{n n} \frac{d z}{d u}
$$

$$
\begin{gathered}
\text { Where, } a_{n 1}=1, \ldots a_{n n}=(n-1) ! y a_{n i}=(n-1) \\
\cdot a_{(n-1)(i-1)}+a_{(n-1) i} \forall n, i=1,2, \ldots, n-1
\end{gathered}
$$

This equation has a method that allows to find the real coefficients a_kj of each term $t^{n} \cdot \frac{d^{n} z}{d t^{n}}$ the equation (1), that after replacing and simplifying there, a homogeneous differential equation of order $\mathrm{n}$ with constant coefficients of the form (2) will be obtained,

$\frac{d^{n} z}{d u^{n}}+c_{n-1} \frac{d^{n-1} z}{d u^{n-1}}+c_{n-2} \frac{d^{n-2} z}{d u^{n-2}}+\ldots+c_{2} \frac{d^{2} z}{d u^{2}}+c_{1} \frac{d z}{d u}+c_{0} z=0$

Now to find all the coefficients $c_{i}$ of the equation (11), can be supported in the equation (10) For the first cases, it means order 1, 2 even up to 3, is usually done directly, but the greater the order, the greater the difficulty in finding the corresponding ODE of type (11) according to. For example, if the Cauchy-Euler equation is of order 3 , according to equations (3) to (9), it is obtained that

$c_{3}=1 ; \quad c_{2}=\left(\mathrm{b}_{2}-3\right) ; \quad c_{1}=\left(\mathrm{b}_{1}-\mathrm{b}_{2}+2\right) ; \quad c_{0}=\mathrm{b}_{0}$

With which the characteristic polynomial corresponding to the homogeneous ODE with constant coefficients associated with the Cauchy-Euler equation

$$
\begin{gathered}
t^{3} z^{(3)}+b_{2} t^{2} z^{(2)}+b_{1} t z^{\prime}+b_{0} z=0 \\
\text { Is, } P_{3}(m)=m^{3}+\left(b_{2}-2\right) m^{2}+\left(b_{1}-b_{2}+2\right) m+b_{0}=0
\end{gathered}
$$

Whose solution is reduced to finding the roots of an algebraic polynomial of degree 3 .

In general, Knowing the characteristic polynomial of (11) for any $n \in \mathbb{N}$,to find the solution of (1), which is the fundamental purpose of this document, would be as simple as finding the solutions of that real polynomial, because if its roots are $r_{1}, r_{2}, \ldots, r_{m}$ with $m \leq n$, then it is a fundamental system of solutions of (11) is,

$$
z_{1}=e^{r_{1} u}, \quad z_{2}=e^{r_{2} u}, \ldots, z_{k}=e^{r_{m} u} \text { con } u=\ln (t)
$$

And therefore, $z_{1}=t^{r_{1}}, z_{2}=t^{r_{2}}, \ldots ., z_{m}=t^{r_{m}}(15)$

Which form a fundamental system of solutions of (3). Now if any $r_{k}=\alpha \pm i \beta$, it means it is a root is complex, then

$$
z_{k}=t^{r_{k}}=\left(e^{\ln (t)}\right)^{r_{k}}=e^{r_{k} \ln (t)}
$$

Of where $z_{k 1}=t^{\alpha} \operatorname{Cos}(\beta \cdot \ln (t)) \quad$ y $\quad z_{k 2}=t^{\alpha} \operatorname{Sen}(\beta \cdot \ln (t))$
On the other hand, if any root $r_{j}$ has multiplicity $k$ then these $k$ solutions would be of the form [4], [5].

$z_{j 1}=t^{r_{j}} \cdot \ln (t), z_{j 2}=t^{r_{j}} \cdot \ln ^{2}(t), \ldots, z_{j k}=t^{r_{j}} \cdot \ln ^{\mathrm{k}}(t)$

Therefore, to find the characteristic polynomial of the differential equation (11) turns out to be fundamental in the solution of (1), for that reason the following Matrix and the following theorem are proposed:

Matrix of the coefficients for the solution of a Cauchy Euler Differential Equation of order n (for the Method proposed in [1])

From equation (10), the square matrix of $n \times n A=\left(a_{j k}\right)$ of the coefficients on the left side of equation (10), where $\mathrm{a}_{\mathrm{jk}}$ is the coefficient of $k-t h$ term in the development of $j-t h$ product $t^{j} \cdot \frac{d^{j} z}{d t^{j}}$ and with $J$ and $k$ varying between 1 ....n. This matrix is unique to each size and is also triangular lower.

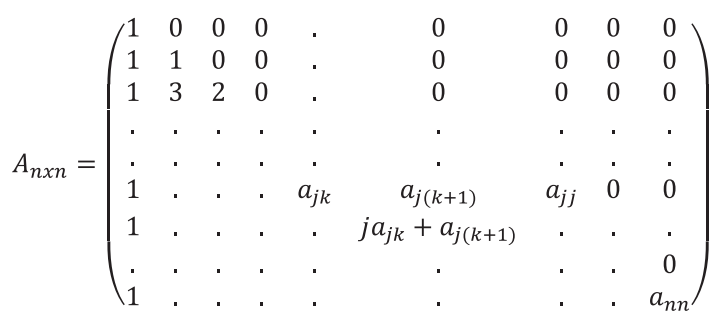

Here it is seen that,

$$
a_{(j+1)(k+1)}=j a_{j k}+a_{j(k+1)}
$$

Here some of them are taken

$$
A_{3 \times 3}=\left(\begin{array}{ccc}
1 & 0 & 0 \\
1 & 1 & 0 \\
1 & 3 & 2
\end{array}\right) A_{4 \times 4}=\left(\begin{array}{cccc}
1 & 0 & 0 & 0 \\
1 & 1 & 0 & 0 \\
1 & 3 & 2 & 0 \\
1 & 6 & 11 & 6
\end{array}\right) \quad A_{6 \times 6}=\left(\begin{array}{cccccc}
1 & 0 & 0 & 0 & 0 & 0 \\
1 & 1 & 0 & 0 & 0 & 0 \\
1 & 3 & 2 & 0 & 0 & 0 \\
1 & 6 & 11 & 6 & 0 & 0 \\
1 & 10 & 35 & 50 & 24 & 0 \\
1 & 15 & 85 & 225 & 274 & 120
\end{array}\right)
$$

Subsequent to the following theorem, we will see the usefulness of matrices with a couple of examples.

Polynomial Theorem Characteristic for solving Cauchy-Euler equations of order $n$ :

It is $\mathrm{t}^{\mathrm{n}} \mathrm{z}^{(\mathrm{n})}+\mathrm{b}_{\mathrm{n}-1} \mathrm{t}^{\mathrm{n}-1} \mathrm{z}^{(\mathrm{n}-1)}+\cdots+\mathrm{b}_{0} \mathrm{z}=0$ a Differential Equation of Cauchy-Euler of order n, whose homogeneous differential equation with associated coefficients constant, is $\frac{d^{n} z}{d u^{n}}+c_{n-1} \frac{d^{n-1} z}{d u^{n-1}}+c_{n-2} \frac{d^{n-2} z}{d u^{n-2}}+\cdots+c_{2} \frac{d^{2} z}{d u^{2}}+c_{1} \frac{d z}{d u}+c_{0} \cdot z=0$ under the substitution $\mathrm{t}=\mathrm{e}^{\mathrm{u}}$ each one of the coefficients $c_{j}$ of the associated ODE of order $n$ can be determined with the equation

$$
c_{j}=\sum_{k=j}^{n}(1)^{k+j} \cdot b_{k} \cdot a_{k(k+1-j)}
$$


Where $\mathrm{a}_{\mathrm{k}(\mathrm{k}+1-\mathrm{j})}$ are the corresponding elements of the matrix (18) of size $n x n$ of Castellanos method in the solution of Cauchy-Euler ODE.

To understand a little more these equations (20) and their use in the solution method of the Cauchy-Euler ODE, a couple of examples are shown

Example 1: we have the following differential equation as a result of an application process

$$
x^{3} y^{\prime \prime \prime}-2 x^{2} y^{\prime \prime}+3 x y^{\prime}-3 y=0
$$

For its solution we see that $b_{3}=1, b_{2}=-2, b_{1}=3$ y $b_{0}=-3$

And in accordance with what is proposed in here, corresponds the matrix

$$
A_{3 \times 3}=\left(\begin{array}{lll}
1 & 0 & 0 \\
1 & 1 & 0 \\
1 & 3 & 2
\end{array}\right)
$$

Now applying the following equations (20) in accordance with the order 3 of this equation (21)

$$
\begin{gathered}
c_{0}=b_{0}=-3 \\
c_{1}=(1)^{1+1} \cdot b_{1} \cdot a_{11}+(1)^{1+2} \cdot b_{2} \cdot a_{22}+(1)^{1+1} \cdot b_{2} \cdot a_{21} \\
c_{1}=3 \cdot 1-(-2) \cdot 1+1 \cdot 2=7 \\
c_{2}=(1)^{2+2} \cdot b_{2} \cdot a_{21}+(1)^{2+3} \cdot b_{3} \cdot a_{32} \\
c_{2}=(-2) \cdot 1-1 \cdot 3=-5 \\
c_{3}=(1)^{3+3} \cdot b_{3} \cdot a_{31}=1 \cdot 1=1
\end{gathered}
$$

It means, $c_{0}=-3, c_{1}=7, c_{2}=-5$ y $c_{3}=1$

Then, the equation with constant coefficients associated, is

$$
\frac{d^{3} y}{d u^{3}}-5 \frac{d^{2} y}{d u^{2}}+7 \frac{d y}{d u}-3 y=0
$$

And its polynomial and characteristic equation is,

$$
\begin{gathered}
P(m)=m^{3}-5 m^{2}+7 m-3=0 \\
P(m)=(m-3)(m-1)(m-1)=0
\end{gathered}
$$

And the roots of this polynomial are, [ $m=1$ ] with multiplicity 2 and [ $m=3$ ] Then, according to the equations (14) to (17)

$$
y_{1}=x^{1} \quad y_{2}=x^{1} \ln (x) \quad y_{3}=x^{3}
$$

Therefore, the solution of (22) is, $y=c_{1} x^{1}+c_{2} x^{1} \ln (x)+c_{3} x^{3}$

Example 2: solve the differential equation

$$
x^{4} y^{(4)}+2 x^{3} y^{(3)}-5 x^{2} y^{(2)}+8 x y^{\prime}-2 y=0
$$

it is observed that $b_{4}=1, b_{3}=2, b_{2}=-5, b_{1}=8$ y $b_{0}=-2$ and in accordance with what is proposed in this document, it corresponds to the matrix

$$
A_{4 \times 4}=\left(\begin{array}{cccc}
1 & 0 & 0 & 0 \\
1 & 1 & 0 & 0 \\
1 & 3 & 2 & 0 \\
1 & 6 & 11 & 6
\end{array}\right)
$$

Now applying the corresponding equations (20) according to order 4 of this equation (22), and described in more detail,

1. $c_{0}=b_{0}=-2$

2. To find $\mathrm{c}_{1}$ we multiply the main diagonal term by term with the elements $b_{1}$ to $b_{4}$, with interspersed signs, starting with positive sign(+), like this,

$$
c_{1}=8 \cdot 1-(-5) \cdot 1+2 \cdot 2-1 \cdot 6=11
$$

3. To find $\mathrm{c}_{2}$ we multiply the diagonal under the main diagonal, I end at term with the elements $\mathrm{b}_{2}$ to $\mathrm{b}_{4}$, with interspersed signs, starting with positive sign(+), like this,

$$
c_{2}=(-5) \cdot 1-3 \cdot 2+1 \cdot 11=0
$$

4. To find $\mathrm{c}_{3}$ we multiply the second diagonal under the main diagonal, $\mathrm{I}$ end at term with the elements $\mathrm{b}_{3}$ to $\mathrm{b}_{4}$ ,with interspersed signs, starting with positive sign(+), like this,

$$
c_{3}=1 \cdot 2-2 \cdot 6=-10
$$

5. To find $\mathrm{c}_{4}$ we multiply the following diagonal, but in this case it is only an element, the $a_{14}$, with the element $\mathrm{b}_{4}$, thus: $c_{4}=1 \cdot 1=1$

Then, the equation with constant coefficients associated with the differential equation (22), is

$$
\frac{d^{4} y}{d u^{4}}-10 \frac{d^{3} y}{d u^{3}}+11 \frac{d y}{d u}-2 y=0
$$

And its polynomial and equation characteristic is,

$P(m)=m^{3}-10 m^{3}+11 m-2=0$

And the roots of this polynomial are,

$m=1, \quad m=9,89, \quad m=0,1877, \quad m=-1,077$

Then, according to the equations (14) to (17)

$y_{1}=x^{1} \quad y_{2}=x^{9,89} \quad y_{1}=x^{0,1877} \quad y_{1}=x^{-1,077}$

Therefore, the solution of (22) is,

$$
y=c_{1} x^{1}+c_{2} x^{9,89}+c_{3} x^{0,1877}+c_{4} \bar{x}^{1,077}
$$

Now, the reader is invited to compare the results of these two examples, with the traditional method shown between equations (3) to (12). 


\section{Conclusions}

-The method shown here to solve differential equations of Cauchy-Euler, is rather functional especially for EDOs of order greater than or equal to 3, because it reduces processes and avoids being making the same differential substitutions over and over again.

-The matrix designed for the solution method of these equations is unique in each size, even the elements of any nxn matrix are the same in any of any larger size, adding to the latter the new elements. This matrix, designed with the algorithm or method proposed in the article (Castellanos et al., 2014), can be seen, since it is triangular inferior, like a triangle of numbers that is always the same, only one more row is added according to the need, similar to what happened with Pascal's triangle.

-With the algorithms or equations proposed in this article and the article [1], a computational template can be easily designed, which allows for any CauchyEuler EDO to solve it by only including the numerical coefficients of the equation, and that delivers the roots of the characteristic polynomial of the ODE with constant coefficients associated with the given Cauchy - Euler, which are the final elements to propose the solution of the equation.

\section{References}

[1] F. H. Castellanos-Moreno and J. F. PantojaBenavides, "A method to determine coefficients in Cauchy-Euler differential equations de order n”, Visión electronica, vol. 7, no. 2, 2014, pp. 6368. https://doi.org/10.14483/22484728.7863.

[2] L. Elsgoltz, "Ecuaciones Diferenciales y Cálculo Variacional”, Moscú: Editorial MIR, 1969, pp. 110-116.

[3] M. Guzmán, "Ecuaciones Diferenciales Ordinarias: Teoría de Estabilidad y Control”, Madrid: Editorial Alhambra, S.A., 1975, pp. 1824.

[4] T. Myint-U and L. Debnath, "Linear Partial Differential Equations for Scientists and Engineers”, Boston-Basel-Berlín: Editorial Birkhäuser, 2007, pp. 1-12.
[5] D. G, Zill, "Ecuaciones Diferenciales con Aplicaciones de Modelado", Cengage Learning Editores S.A., 2009, Novena Edición, pp. 162167. 